\title{
Vortex line in spin-orbit coupled atomic Fermi gases
}

\author{
M. Iskin \\ Department of Physics, Koç University, Rumelifeneri Yolu, TR-34450 Sariyer, Istanbul, Turkey
}

(Received 1 December 2011; published 17 January 2012)

\begin{abstract}
It has recently been shown that the spin-orbit coupling gives rise to topologically nontrivial and thermodynamically stable gapless superfluid phases when the pseudospin populations of an atomic Fermi gas are imbalanced, with the possibility of featuring Majorana zero-energy quasiparticles. In this paper, we consider a Rashba-type spin-orbit coupling, and use the Bogoliubov-de Gennes formalism to analyze a single vortex line along a finite cylinder with a periodic boundary condition. We show that the signatures for the appearance of coreand edge-bound states can be directly found in the density of single-particle states and particle-current density. In particular, we find that the pseudospin components counterflow near the edge of the cylinder, the strength of which increases with increasing spin-orbit coupling.
\end{abstract}

DOI: 10.1103/PhysRevA.85.013622

PACS number(s): 03.75.Ss, 05.30.Fk, 03.75.Hh

\section{INTRODUCTION}

Following the recent experimental success with artificial gauge fields and spin-orbit coupled atomic Bose gases [1,2], there has been increasing theoretical interest in studying spin-orbit coupled atomic Fermi gases with balanced or imbalanced populations, at zero or finite temperatures, in two or three dimensions, etc. [3-16]. The main motivation behind these works is that the spin-orbit coupled atomic Fermi gases are ideal systems for studying topologically nontrivial superfluid phases [3,7-9], with the possibility of featuring Majorana zero-energy bound states for which the associated quasiparticle operators are self-Hermitian. This means that a zero-energy Majorana quasiparticle is its own antiquasiparticle. Although these quasiparticles are predicted to appear in low-dimensional strongly correlated systems in various fields of physics, including the fractional quantum Hall systems [17], chiral two-dimensional superconductors $[18,19]$, chiral two-dimensional $p$-wave superfluids [20,21], three-dimensional topological insulator-superconductor heterostructures [22], one-dimensional nanowires [23,24], spinorbit coupled semiconductor-superconductor heterostructures $[25,26]$, etc., it has proven to be very difficult to realize them in these systems. Given that the cold-atom systems offer unprecedented control in comparison to condensed-matter ones, there is a good chance of creating and observing Majorana quasiparticles with atomic systems in the near future.

The first step in searching for the Majorana quasiparticles with spin-orbit coupled Fermi gases is to understand the phase diagram of these systems, which has recently been worked out within the mean-field approximation [3,7-9]. For instance, the ground-state phase diagram of a Rashba-type spin-orbit coupling at unitarity, i.e., when the two-body scattering length $a_{s}$ between pseudospin components in vacuum diverges, is illustrated in Fig. 1. There are three phases in the phase diagram [7-9]. While the normal (N) phase is characterized by a vanishing superfluid order parameter, the uniform superfluid and nonuniform superfluid, e.g., phase separation (PS), are distinguished by their thermodynamic stability when the order parameter is nonzero. Furthermore, in addition to the topologically trivial gapped superfluid (SF) phase, the gapless superfluid (GSF) phase can be distinguished by the momentum-space topology of its excitations. Depending on the number of zero-quasiparticle excitation energy regions in momentum space, there are two topologically distinct gapless phases. For the Rashba-type spin-orbit coupling shown here, while GSF(II) has four zero-energy points, GSF(I) has only two.

The phase diagram illustrates that the spin-orbit coupling counteracts the population imbalance, and that this competition tends to stabilize the GSF phase against PS. The anisotropic nature of the spin-orbit coupling (in momentum space) is also found to stabilize exotic superfluid phases. For instance, in sharp contrast to the $\alpha=0$ case where only the gapless superfluid phase supports population imbalance, both the gapless and gapped superfluid phases are found to support population imbalance. Although Rashba-type spinorbit coupling is considered in Fig. 1, the topological structure shown here is quite robust against the effects of anisotropic spin-orbit couplings [7].

Since Majorana quasiparticles appear in the presence of topological defects, e.g., vortices, domain walls, boundaries between bulk phases, etc., one of the exciting experimental directions with spin-orbit coupled Fermi gases may be to create and observe a Majorana zero-energy quasiparticle bound to a vortex. For this purpose, in this paper, we consider a Rashba-type spin-orbit coupling, and use the Bogoliubov-de Gennes $(\mathrm{BdG})$ formalism to analyze a single vortex line along a finite cylinder with a periodic boundary condition. We find signatures for the appearance of core- and edge-bound states in various observables, most notable of which is the counterflow of pseudospin particle-current densities near the edge of the cylinder. The rest of the paper is organized as follows. First, we generalize the BdG formalism to spin-orbit coupled Fermi gases in Sec. II, and then derive the selfconsistency equations for a single vortex line in Sec. III. We present the numerical solutions in Sec. IV, where we discuss the effects of spin-orbit coupling on the superfluid order parameter, particle density, energy spectrum, bound-state wave functions, single-particle density of states, and probabilitycurrent density. Our conclusions are briefly summarized in Sec. V. 


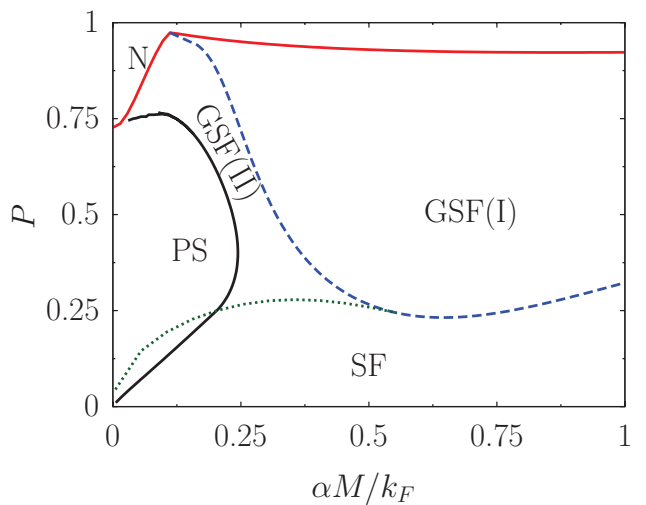

FIG. 1. (Color online) The mean-field ground-state phase diagram of a Rashba-type spin-orbit coupled Fermi gas is shown as a function of the population-imbalance parameter $P=\left(N_{\uparrow}-N_{\downarrow}\right) / N$ and the strength of the spin-orbit coupling $\alpha$ at unitarity, i.e., when $\left|a_{s}\right| \rightarrow \infty$. Here, N, SF, GSF, and PS correspond to normal phase, topologically trivial gapped superfluid, topologically nontrivial gapless superfluid, and phase separation, respectively. (Adapted from Ref. [7].)

\section{BOGOLIUBOV-DE GENNES FORMALISM}

The results mentioned above are obtained by solving the self-consistent BdG equations, suitably generalized to spinorbit coupled Fermi gases. Before presenting our numerical results, let us first present the theoretical framework of this formalism.

\section{A. Hamiltonian}

In this paper, we use the mean-field Hamiltonian density (in units of $\left.\hbar=k_{B}=1\right), \quad H(\mathbf{r})=\sum_{\sigma, \sigma^{\prime}} \psi_{\sigma}^{\dagger}(\mathbf{r}) K_{\sigma \sigma^{\prime}}(\mathbf{r}) \psi_{\sigma^{\prime}}(\mathbf{r})+$ $\Delta(\mathbf{r}) \psi_{\uparrow}^{\dagger}(\mathbf{r}) \psi_{\downarrow}^{\dagger}(\mathbf{r})+\Delta^{*}(\mathbf{r}) \psi_{\downarrow}(\mathbf{r}) \psi_{\uparrow}(\mathbf{r}), \quad$ describing twocomponent Fermi gases with attractive and short-range interactions. Here, the operators $\psi_{\sigma}^{\dagger}(\mathbf{r})$ and $\psi_{\sigma}(\mathbf{r})$ create and annihilate a pseudospin $\sigma$ fermion at position $\mathbf{r}$, respectively, and $\Delta(\mathbf{r})$ is the mean-field superfluid order parameter. Furthermore, the operator $K_{\sigma \sigma}(\mathbf{r})=-\nabla^{2} /\left(2 M_{\sigma}\right)-\mu_{\sigma}$ is the kinetic energy, where $M_{\sigma}$ is the mass and $\mu_{\sigma}$ is the chemical potential of $\sigma$ fermions, and the operator $K_{\uparrow \downarrow}(\mathbf{r})=K_{\downarrow \uparrow}^{\dagger}(\mathbf{r})=\alpha\left(p_{y}+i p_{x}\right)$ is the spin-orbit coupling, where $\alpha \geqslant 0$ is its strength and $p_{j}=-i \partial / \partial j$ is the momentum operator. Although we keep the formalism quite general, we present numerical calculations only for the mass-balanced Fermi gases with $M_{\uparrow}=M_{\downarrow}=M$.

In the presence of a spin-orbit coupling, this Hamiltonian can be diagonalized via the generalized Bogoliubov-Valatin transformations, $\psi_{\sigma}(\mathbf{r})=\sum_{n, \sigma^{\prime}}\left[u_{n}^{\sigma \sigma^{\prime}}(\mathbf{r}) \gamma_{n, \sigma^{\prime}}+v_{n}^{\sigma \sigma^{\prime *}}(\mathbf{r}) \gamma_{n, \sigma^{\prime}}^{\dagger}\right]$, where $u_{n}^{\sigma \sigma^{\prime}}(\mathbf{r})$ and $v_{n}^{\sigma \sigma^{\prime}}(\mathbf{r})$ are the amplitudes, and $\gamma_{n, \sigma}^{\dagger}$ and $\gamma_{n, \sigma}$ are the operators corresponding to the creation and annihilation of pseudospin $\sigma$ quasiparticles, respectively. The resultant BdG equation can be written as $H(\mathbf{r}) \varphi_{n}^{\sigma^{\prime}}(\mathbf{r})=\varepsilon_{n}^{\sigma^{\prime}} \varphi_{n}^{\sigma^{\prime}}(\mathbf{r})$, where

$$
H(\mathbf{r})=\left[\begin{array}{cccc}
K_{\uparrow \uparrow}(\mathbf{r}) & K_{\uparrow \downarrow}(\mathbf{r}) & 0 & \Delta(\mathbf{r}) \\
K_{\downarrow \uparrow}(\mathbf{r}) & K_{\downarrow \downarrow}(\mathbf{r}) & -\Delta(\mathbf{r}) & 0 \\
0 & -\Delta^{*}(\mathbf{r}) & -K_{\uparrow \uparrow}^{*}(\mathbf{r}) & -K_{\uparrow \downarrow}^{*}(\mathbf{r}) \\
\Delta^{*}(\mathbf{r}) & 0 & -K_{\downarrow \uparrow}^{*}(\mathbf{r}) & -K_{\downarrow \downarrow}^{*}(\mathbf{r})
\end{array}\right]
$$

is the Hamiltonian matrix given in the $\varphi_{n}^{\sigma^{\prime}}(\mathbf{r})=$ $\left[u_{n}^{\uparrow \sigma^{\prime}}(\mathbf{r}), u_{n}^{\downarrow \sigma^{\prime}}(\mathbf{r}), v_{n}^{\uparrow \sigma^{\prime}}(\mathbf{r}), v_{n}^{\downarrow \sigma^{\prime}}(\mathbf{r})\right]^{T}$ basis, and $\varepsilon_{n}^{\sigma^{\prime}} \geqslant 0$ are the energy eigenvalues. Since the BdG equations do not depend on $\sigma^{\prime}$, it is sufficient to solve only for $u_{n}^{\sigma}(\mathbf{r})=u_{n}^{\sigma \sigma^{\prime}}(\mathbf{r})$, $v_{n}^{\sigma}(\mathbf{r})=v_{n}^{\sigma \sigma^{\prime}}(\mathbf{r})$, and $\varepsilon_{n}=\varepsilon_{n}^{\sigma^{\prime}}$.

\section{B. Self-consistency equations}

Using the Bogoliubov-Valatin transformations, the meanfield superfluid order parameter $\Delta(\mathbf{r})=g\left\langle\psi_{\uparrow}(\mathbf{r}) \psi_{\downarrow}(\mathbf{r})\right\rangle$, where $g \geqslant 0$ is the strength of the attractive interaction between $\uparrow$ and $\downarrow$ fermions, and $\langle\cdots\rangle$ is the thermal average, becomes $\Delta(\mathbf{r})=$ $g \sum_{n}\left[u_{n}^{\uparrow}(\mathbf{r}) v_{n}^{\downarrow *}(\mathbf{r}) f\left(\varepsilon_{n}\right)+u_{n}^{\downarrow}(\mathbf{r}) v_{n}^{\uparrow *}(\mathbf{r}) f\left(-\varepsilon_{n}\right)\right]$. Here, $f(x)=$ $1 /\left(e^{x / T}+1\right)$ is the Fermi function and $T$ is the temperature. As usual, we relate the interaction strength $g$ to the two-body scattering length $a_{s}$ between an $\uparrow$ and a $\downarrow$ fermion in vacuum via the relation $1 / g=-M_{r} V /\left(4 \pi a_{s}\right)+\sum_{\mathbf{k}} 1 /\left(\varepsilon_{\mathbf{k}, \uparrow}+\varepsilon_{\mathbf{k}, \downarrow}\right)$, where $M_{r}=2 M_{\uparrow} M_{\downarrow} /\left(M_{\uparrow}+M_{\downarrow}\right)$ is twice the reduced mass of an $\uparrow$ and a $\downarrow$ fermion, $V$ is the volume of the sample, and $\varepsilon_{\mathbf{k}, \sigma}=k^{2} /\left(2 M_{\sigma}\right)$ is the kinetic energy. This leads to $g=4 \pi^{2} a_{s} /\left[2 M_{r} a_{s} \sqrt{2 M_{r} \varepsilon_{c}}-M_{r} \pi\right]$, where $\varepsilon_{c}$ is the energy cutoff used in the $\mathbf{k}$-space integration (to be specified below in Sec. IV).

To determine $\mu_{\sigma}$, the order-parameter equation has to be solved self-consistently with the number equations $N_{\sigma}=$ $\int d \mathbf{r} n_{\sigma}(\mathbf{r})$, where $n_{\sigma}(\mathbf{r})=\left\langle\psi_{\sigma}^{\dagger}(\mathbf{r}) \psi_{\sigma}(\mathbf{r})\right\rangle$ is the local density of $\sigma$ fermions. Using the Bogoliubov-Valatin transformations, we obtain $n_{\sigma}(\mathbf{r})=\sum_{n}\left[\left|u_{n}^{\sigma}(\mathbf{r})\right|^{2} f\left(\varepsilon_{n}\right)+\left|v_{n}^{\sigma}(\mathbf{r})\right|^{2} f\left(-\varepsilon_{n}\right)\right]$. Having generalized the $\mathrm{BdG}$ formalism to spin-orbit coupled Fermi gases, next we apply it for a single vortex line.

\section{SINGLE VORTEX LINE}

In particular, we consider a single vortex line positioned along a finite cylinder of radius $R$ and length $L$, and with a periodic boundary condition in the $z$ direction, in such a way that the order parameter can be written as $\Delta(\mathbf{r})=\Delta(r) e^{-i \theta}$, where $r$ and $\theta$ are the cylindrical coordinates $\mathbf{r}=(r, \theta, z)$ [27]. Note in this coordinate system that the spin-orbit coupling term becomes $K_{\uparrow \downarrow}(\mathbf{r})=e^{-i \theta}[\partial / \partial r-(i / r) \partial / \partial \theta]$, showing that the single vortex line has rotational invariance around the $z$ axis, so that the solutions of the BdG equation have a well-defined planar angular momentum $m$, i.e., $m$ is a good quantum number. In addition, the system is assumed to have translational invariance along the $z$ direction, i.e., $p_{z}$ momentum is also a good quantum number.

Thus, for a singly-quantized vortex line considered in this paper, we may choose the normalized wave functions as $u_{n}^{\uparrow}(\mathbf{r})=u_{n m s}^{\uparrow}(r) e^{i m \theta} e^{i k_{s} z} / \sqrt{2 \pi L}$ and $v_{n}^{\uparrow}(\mathbf{r})=v_{n m s}^{\uparrow}(r) e^{i(m+2) \theta} e^{i k_{s} z} / \sqrt{2 \pi L}$ for the $\uparrow$ components, and $u_{n}^{\downarrow}(\mathbf{r})=u_{n m s}^{\downarrow}(r) e^{i(m+1) \theta} e^{i k_{s} z} / \sqrt{2 \pi L}$ and $v_{n}^{\downarrow}(\mathbf{r})=$ $v_{n m s}^{\downarrow}(r) e^{i(m+1) \theta} e^{i k_{s} z} / \sqrt{2 \pi L}$ for the $\downarrow$ ones. Here, $k_{s}=2 \pi s / L$ is the wave vector along the $z$ direction with $s=0, \pm 1$, $\pm 2, \ldots$. This particular choice (which is not unique $[25,26]$ ) allows us to decouple the $\mathrm{BdG}$ equations into independent subspaces of $(m, s)$ sectors. We further project the radial wave functions onto a set of Bessel functions normalized in a disk of radius $R$ [27], i.e., $\phi_{j, m}(r)=\sqrt{2} J_{m}\left(\beta_{j, m} r / R\right) /\left[R J_{m+1}\left(\beta_{j, m}\right)\right]$, where $j=1,2,3, \ldots$ and the argument $\beta_{j, m}$ is the $j$ th zero of 
$J_{m}(x)$. More explicitly, we have $u_{n m s}^{\uparrow}(r)=\sum_{j} c_{n m s j}^{\uparrow} \phi_{j, m}(r)$ and $v_{n m s}^{\uparrow}(r)=\sum_{j} d_{n m s j}^{\uparrow} \phi_{j, m+2}(r)$ for the $\uparrow$ radial wave functions, and $u_{n m s}^{\downarrow}(r)=\sum_{j} c_{n m s j}^{\downarrow} \phi_{j, m+1}(r)$ and $v_{n m s}^{\downarrow}(r)=$ $\sum_{j} d_{n m s j}^{\downarrow} \phi_{j, m+1}(r)$ for the $\downarrow$ ones, and they already satisfy the boundary conditions $u_{n m s}^{\sigma}(R)=v_{n m s}^{\sigma}(R)=0$ at the edge of the cylinder.

Using the orthonormality condition $\int_{0}^{R} r d r \phi_{j, m}(r) \phi_{j^{\prime}, m}$ $(r)=\delta_{j j^{\prime}}$ where $\delta_{j j^{\prime}}$ is the Kronecker delta, this procedure reduces the $\mathrm{BdG}$ equation given in Eq. (1) to a $4 j_{\max } \times 4 j_{\max }$ matrix eigenvalue problem,

$$
\begin{aligned}
& \sum_{j^{\prime}}\left(\begin{array}{cccc}
K_{\uparrow, m s}^{j j^{\prime}} & S_{m}^{j j^{\prime}} & 0 & \Delta_{m}^{j j^{\prime}} \\
S_{m}^{j j^{\prime}} & K_{\downarrow, m s}^{j j^{\prime}} & -\Delta_{m+1}^{j j^{\prime}} & 0 \\
0 & -\Delta_{m+1}^{j j^{\prime}} & -K_{\uparrow, m s}^{j j^{\prime}} & S_{m+1}^{j^{\prime} j} \\
\Delta_{m}^{j j^{\prime}} & 0 & S_{m+1}^{j^{\prime} j} & -K_{\downarrow, m s}^{j j^{\prime}}
\end{array}\right)\left(\begin{array}{l}
c_{n m s j^{\prime}}^{\uparrow} \\
c_{n m s j^{\prime}}^{\downarrow} \\
d_{n m s j^{\prime}}^{\uparrow} \\
d_{n m s j^{\prime}}^{\downarrow}
\end{array}\right) \\
& =\varepsilon_{n m s}\left(\begin{array}{c}
c_{n m s j}^{\uparrow} \\
c_{n m s j}^{\downarrow} \\
d_{n m s j}^{\uparrow} \\
d_{n m s j}^{\downarrow}
\end{array}\right),
\end{aligned}
$$

for each $(m, s)$ sector, if we allow $1 \leqslant j \leqslant j_{\max }$ states. Here, $\quad K_{\sigma, m s}^{j j^{\prime}}=\left[\beta_{j, m}^{2} /\left(2 M_{\sigma} R^{2}\right)+k_{s}^{2} /\left(2 M_{\sigma}\right)-\mu_{\sigma}\right] \delta_{j j^{\prime}} \quad$ are the kinetic energy terms, $S_{m}^{j j^{\prime}}=\alpha \int_{0}^{R} r d r \phi_{j, m}(r)[\partial / \partial r+$ $(m+1) / r] \phi_{j^{\prime}, m+1}(r)$ are the spin-orbit coupling terms leading to $S_{m}^{j j^{\prime}}=\alpha C_{j^{\prime} m} \int_{0}^{R} r d r \phi_{j, m}(r) J_{m}\left(\beta_{j^{\prime}, m+1} r / R\right)$ where $C_{j^{\prime} m}=\sqrt{2} \beta_{j^{\prime}, m+1} /\left[R^{2} J_{m+2}\left(\beta_{j^{\prime}, m+1}\right)\right]$, and $\Delta_{m}^{j j^{\prime}}=$ $\int_{0}^{R} r d r \Delta(r) \phi_{j, m}(r) \phi_{j^{\prime}, m+1}(r)$ are the pairing terms. The same procedure also reduces the order-parameter equation to

$$
\begin{aligned}
\Delta(r)= & \frac{g}{2 \pi L} \sum_{n m s j j^{\prime}}\left[c_{n m s j}^{\downarrow} d_{n m s j^{\prime}}^{\uparrow} \phi_{j, m+1}(r) \phi_{j^{\prime}, m+2}(r) f\left(\varepsilon_{n m s}\right)\right. \\
& \left.+c_{n m s j}^{\uparrow} d_{n m s j^{\prime}}^{\downarrow} \phi_{j, m}(r) \phi_{j^{\prime}, m+1}(r) f\left(-\varepsilon_{n m s}\right)\right]
\end{aligned}
$$

and the local-density equations to

$$
\begin{aligned}
n_{\uparrow}(r)= & \frac{1}{2 \pi L} \sum_{n m s j j^{\prime}}\left[c_{n m s j}^{\uparrow} c_{n m s j^{\prime}}^{\uparrow} \phi_{j, m}(r) \phi_{j^{\prime}, m}(r) f\left(\varepsilon_{n m s}\right)\right. \\
& \left.+d_{n m s j}^{\uparrow} d_{n m s j^{\prime}}^{\uparrow} \phi_{j, m+1}(r) \phi_{j^{\prime}, m+2}(r) f\left(-\varepsilon_{n m s}\right)\right] \\
n_{\downarrow}(r)= & \frac{1}{2 \pi L} \sum_{n m s j j^{\prime}}\left[c_{n m s j}^{\downarrow} c_{n m s j^{\prime}}^{\downarrow} \phi_{j, m+1}(r) \phi_{j^{\prime}, m+1}(r) f\left(\varepsilon_{n m s}\right)\right. \\
& \left.+d_{n m s j}^{\downarrow} d_{n m s j^{\prime}}^{\downarrow} \phi_{j, m+1}(r) \phi_{j^{\prime}, m+1}(r) f\left(-\varepsilon_{n m s}\right)\right] .
\end{aligned}
$$

We recall that the sums are only over the quasiparticle states with $\varepsilon_{n m s} \geqslant 0$. Using the orthonormality condition, we also obtain the total number of $\sigma$ fermions as $N_{\sigma}=$ $\sum_{n m s j}\left[\left(c_{n m s j}^{\sigma}\right)^{2} f\left(\varepsilon_{n m s}\right)+\left(d_{n m s j}^{\sigma}\right)^{2} f\left(-\varepsilon_{n m s}\right)\right]$. We emphasize that these mean-field equations can be used for all values of $a_{s}$ and $\alpha$ at low $T$, but they provide only a qualitative description of the system outside of the weak-coupling regime, i.e., in the BCS-BEC crossover. In this paper, we set the temperature to zero, and consider a strongly interacting Fermi gas at unitarity, i.e., $\left|a_{s}\right| \rightarrow \infty$, as a function of $\alpha$.

\section{NUMERICAL RESULTS}

In our numerical calculations, we set a large energy cutoff $\varepsilon_{c}=10 \varepsilon_{F}$, and numerically solve the self-consistency Eqs. (2)-(5) at $T=0$. Here, $\varepsilon_{F}=k_{F}^{2} /(2 M)$ is a characteristic Fermi-energy scale where $k_{F}$ is the Fermi momentum corresponding to the bulk value of the total density of fermions, i.e., $n_{\uparrow}(r)+n_{\downarrow}(r)=k_{F}^{3} /\left(3 \pi^{2}\right)$ at the bulk. We also choose $R=25 / k_{F}$ as the radius and $L=10 / k_{F}$ as the length of the cylinder, and $j_{\max }=50$ and $|m|_{\max }=75$ as the maximum quantum numbers. Note that $|s|_{\max }=L \sqrt{M \varepsilon_{c} / 2} / \pi$, in order to be consistent with the energy cutoff. Since the presence of a single vortex line cannot significantly affect the bulk parameters, we first solve $\mu_{\sigma}$ and $\Delta_{0}$ self-consistently for a vortex-free thermodynamic system, and then use these solutions as an input for our vortex-line calculation, where $\Delta_{0}$ corresponds to the bulk value of $\Delta(r)$. Here, we assume $\Delta(r)$ is real without losing generality.

\section{A. Order parameter and density of fermions}

In Fig. 2(a), we show typical order-parameter profiles $\Delta(r)$ for $\alpha=0.5 k_{F} / M$ and $\alpha=k_{F} / M$ when $P=0.5$, i.e., it rapidly increases from zero around the vortex core, saturates to its bulk value $\Delta_{0}$ around $k_{F} r \simeq 5$, and then rapidly decreases to zero near the edge of the cylinder [27]. Here, the populationimbalance parameter $P=\left[n_{\uparrow}(r)-n_{\downarrow}(r)\right] /\left[n_{\uparrow}(r)+n_{\downarrow}(r)\right]$ is defined at the bulk. We see that $\Delta(r)$ increases with increasing $\alpha$, e.g., its bulk value increases from $0.50 \varepsilon_{F}$ to $0.66 \varepsilon_{F}$, and that the effect of spin-orbit coupling is similar to the effect of increased interaction strength. This is due to the increased

(a)

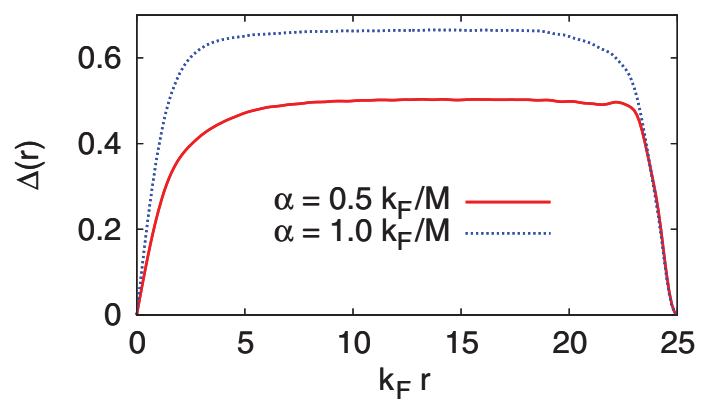

(b)

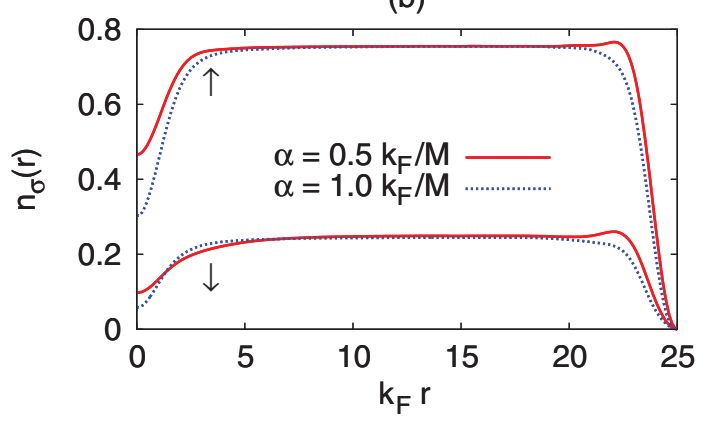

FIG. 2. (Color online) The order parameter $\Delta(r)$ (in units of $\left.\varepsilon_{F}\right)$ and density $n_{\sigma}(r)$ [in units of $k_{F}^{3} /\left(3 \pi^{2}\right)$ ] profiles are shown as a function of radial distance $r$ (in units of $1 / k_{F}$ ). Here, we set $1 /\left(k_{F} a_{s}\right)=0$ and $P=0.5$, and vary $\alpha$. 
density of states with increasing $\alpha$, and it is consistent with the previous results on thermodynamic systems [3-16].

In Fig. 2(b), we show the corresponding density profiles $n_{\sigma}(r)$ for the same parameters. When $\alpha=0$, it is well known for the fermion superfluids that the density is depleted near the vortex core compared to its bulk value [27], and that the depletion increases with increasing interaction strength toward the molecular BEC side. This is because the energy separation between the vortex core-bound states increases with increasing $\Delta_{0}$, which makes them less occupied. In fact, for a population-balanced Fermi gas, the density depletes fully and becomes zero at the vortex core in the molecular BEC limit, consistent with the theory of weakly interacting atomic Bose gases. However, for population-imbalanced Fermi gases, the vortex core may still be filled with excess fermions toward this limit [28]. When $\alpha \neq 0$, in Fig. 2(b), we again see that the effect of spin-orbit coupling is similar to the effect of increased interaction strength, i.e., density depletion also increases for both $\sigma$ components with increasing $\alpha$. To further understand the density depletions, next we analyze the spectrum of energy eigenvalues.

\section{B. Energy spectrum}

In Fig. 3, the spectra of energy eigenvalues $\varepsilon_{n m s}$ are shown as a function of planar angular momentum $m$ for the $s=0$ sector. The spectrum rapidly becomes symmetric around $m=0$ with increasing $s$, since the vortex core and

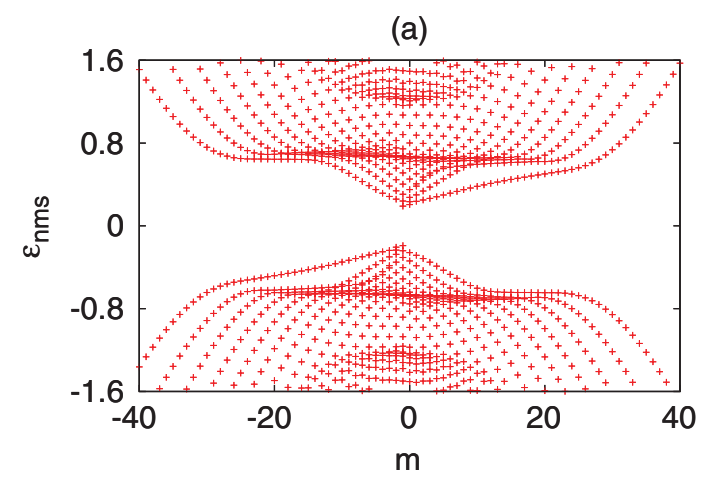

(b)

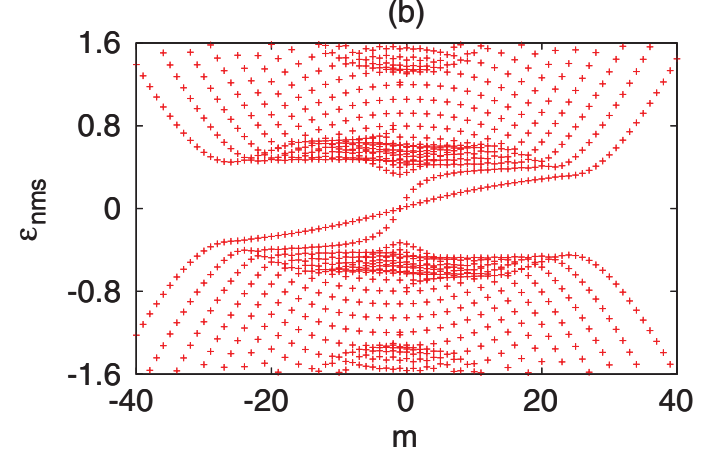

FIG. 3. (Color online) The energy spectrum $\varepsilon_{n m s}$ (in units of $\varepsilon_{F}$ ) is shown as a function of planar angular momentum $m$ when $s=$ 0 . Here, we set $1 /\left(k_{F} a_{s}\right)=0$ and $\alpha=0.5 k_{F} / M$, and vary $P$. In (a) we choose $P=0.2$ corresponding to a topologically trivial gapped bulk SF phase, and in (b) we choose $P=0.5$ corresponding to a topologically nontrivial gapless bulk GSF phase. The linear branch in (b) corresponds to the edge-bound states. edge states disappear when $|s| \sim 1$. Here, we choose $\alpha$ and $P$ such that Fig. 3(a) corresponds to a topologically trivial gapped bulk SF phase, and Fig. 3(b) corresponds to a topologically nontrivial gapless bulk GSF phase (see the thermodynamic phase diagram given in Fig. 1). First of all, we note that the excitation spectra shown in these figures have the necessary symmetry, $\varepsilon_{n m s}=-\varepsilon_{n,-(m+2), s}$, which follows from the particle-hole symmetry of the Hamiltonian. In addition, a second branch of continuum spectra appears in both cases and on both positive and negative energy regions when $\left|\varepsilon_{n m s}\right| \gtrsim 1.2 \varepsilon_{F}$. This is similar to what happens in a thermodynamic system, for which the excitation spectrum has two quasiparticle and two quasihole branches when $\alpha \neq 0[7,9]$.

When $\alpha=0$, the positive- and negative-energy spectra are connected by a single branch of discrete Andreev-like bound states [27,29]. While the visible discreteness of the continuum spectrum is a finite-size effect and the spectrum becomes continuous only in the thermodynamic limit $\left(k_{F} R \rightarrow \infty\right)$, the discreteness of the bound states is insensitive to the system size since these states are strongly localized around the vortex core. The lowest-energy quasiparticle excitation requires a minigap of the order $\Delta_{0}^{2} /\left(2 \varepsilon_{F}\right) \ll \Delta_{0}$. When $\alpha \neq 0$, the energy spectrum of the SF phase shown in Fig. 3(a) is very similar to the usual population-balanced $s$-wave superfluids, for which the bulk energy spectrum is also gapped. There are only a few discrete core-bound states appearing within the bulk energy gap in Fig. 3(a), since the bulk order parameter $\Delta_{0} \simeq 0.7 \varepsilon_{F}$ is quite large for $P=0.2$, leading also to a large minigap. However, the bulk order parameter decreases to $\Delta_{0} \simeq 0.5 \varepsilon_{F}$ when $P=0.5$, leading to a smaller minigap in comparison to the $P=0.2$ case, and hence a larger number of core-bound states, as can be clearly seen in Fig. 3(b).

In contrast, we see a major difference in the energy spectrum of the GSF phase, as shown in Fig. 3(b). In addition to the branch of discrete core-bound states that is also present in the SF phase, there is a second branch of bound states which are strongly localized around the edge of the cylinder. These states result from Andreev scattering at the rigid walls of the cylinder, and their spectrum is linear in energy within the continuum gap [30]. We find that the lowest positive-energy and highest negative-energy bound states have $m=-1$ and $s=0$, and their energies are $\varepsilon_{0} \approx 4.33 \times 10^{-4} \varepsilon_{F}$ and $\varepsilon_{0^{\prime}} \approx$ $-4.33 \times 10^{-4} \varepsilon_{F}$, respectively. This is not a coincidence, since we know that the energy spectrum has $\varepsilon_{n m s}=-\varepsilon_{n,-(m+2), s}$ symmetry; and given that the spectrum is expected to have a twofold degenerate zero-energy bound state, i.e., a pair of Majorana quasiparticles, in the thermodynamic limit, they must occur at $m=-1$. However, hybridization between the core- and edge-bound states (see below) lifts this degeneracy in a finite system, and the zero-energy bound states split in energy, as we find here.

In Fig. 4, the radial wave functions $u_{n m s}^{\sigma}(r)$ and $v_{n m s}^{\sigma}(r)$ of the lowest positive-energy bound state with energy $\varepsilon_{0}$ are shown as a function of radial distance $r$, for the parameters considered in Fig. 3(b). [Due to particle-hole symmetry, the radial wave functions for the highest negative-energy bound state with energy $\varepsilon_{0^{\prime}}$ can simply be obtained by changing $u_{n m s}^{\sigma}(r) \rightarrow v_{n m s}^{\sigma}(r)$, and vice versa.] We note that while the wave functions have $u_{n m s}^{\sigma}(r)=-v_{n m s}^{\sigma}(r)$ symmetry near 


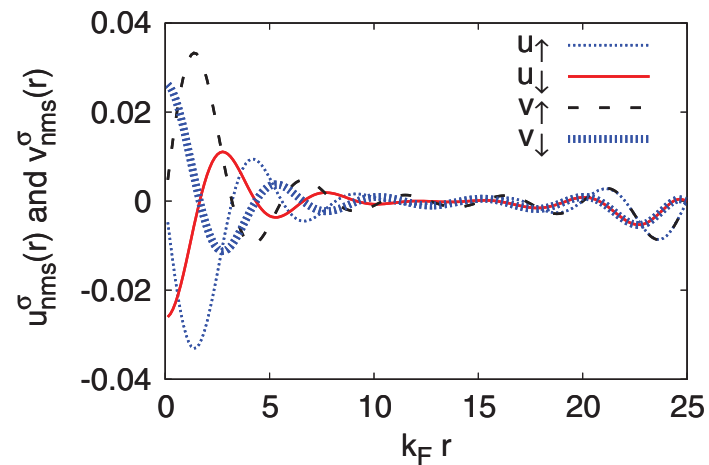

FIG. 4. (Color online) The radial wave functions $u_{n m s}^{\sigma}(r)$ and $v_{n m s}^{\sigma}(r)$ (in units of $1 / k_{F}$ ) are shown as a function of radial distance $r$ (in units of $1 / k_{F}$ ) for the lowest positive-energy bound state. For the parameters considered in Fig. $3(\mathrm{~b}), \varepsilon_{0} \approx 4.33 \times 10^{-4} \varepsilon_{F}$, and it occurs at $m=-1$ and $s=0$.

the vortex core, they have $u_{n m s}^{\sigma}(r)=v_{n m s}^{\sigma}(r)$ symmetry near the edge. These are consistent with the symmetries of Majorana quasiparticles [26], for which the associated quasiparticle operators are self-Hermitian, i.e., a Majorana quasiparticle is its own antiquasiparticle. This is clearly seen from the Bogoliubov-Valatin quasiparticle creation operator $\gamma_{n}^{\dagger}=$ $\int d \mathbf{r} \sum_{\sigma}\left[u_{n}^{\sigma}(\mathbf{r}) \psi_{\sigma}^{\dagger}(\mathbf{r})+v_{n}^{\sigma}(\mathbf{r}) \psi_{\sigma}(\mathbf{r})\right]$ evaluated at $m=-1$ and $s=0$. Since the Majorana quasiparticles always come in pairs, they appear simultaneously but away from each other in real space. In our single vortex line, while one of them is mostly localized at the vortex core, the other one is mostly localized at the edge, with some degree of hybridization between them due to finite-size effects. The hybridization is clearly seen in the wave functions shown in Fig. 4. We note that due to this coupling between the Majorana core- and edge-bound states, their twofold $\varepsilon_{n m s}=0$ degeneracy is lifted, causing a small level splitting, as discussed above. Increasing the separation between Majorana core- and edge-bound states, i.e., when $k_{F} R \rightarrow \infty$, weakens the hybridization such that both bound states eventually become degenerate in energy with $\varepsilon_{n m s}=0$. When this happens, the core quasiparticle is well localized around the vortex core with $u_{n m s}^{\sigma}(r)=-v_{n m s}^{\sigma}(r)$ symmetry, and the edge quasiparticle is well localized around the edge with $u_{n m s}^{\sigma}(r)=v_{n m s}^{\sigma}(r)$ symmetry, without any hybridization between the two.

So far, we have established a major difference between the energy spectra of SF and GSF phases, which is mainly due to the appearance of edge-bound and Majorana zero-energy bound states, and this difference leaves its signatures in various observables, as discussed next.

\section{Single-particle density of states}

For instance, the local single-particle density of $\sigma$ states $D_{\sigma}(\mathbf{r}, \omega)=\sum_{n}\left[\left|u_{n}^{\sigma}(\mathbf{r})\right|^{2} \delta\left(\omega-\varepsilon_{n}\right)+\left|v_{n}^{\sigma}(\mathbf{r})\right|^{2} \delta(\omega+\right.$ $\left.\varepsilon_{n}\right)$ ], where $\delta(x)$ is the delta function, as well as the integrated single-particle density of $\sigma$ states $D_{\sigma}(\omega)=\int d \mathbf{r} D_{\sigma}(\mathbf{r}, \omega)$, provide direct evidence for the existence of edge-bound and Majorana zero-energy bound states, as shown below. In (a)

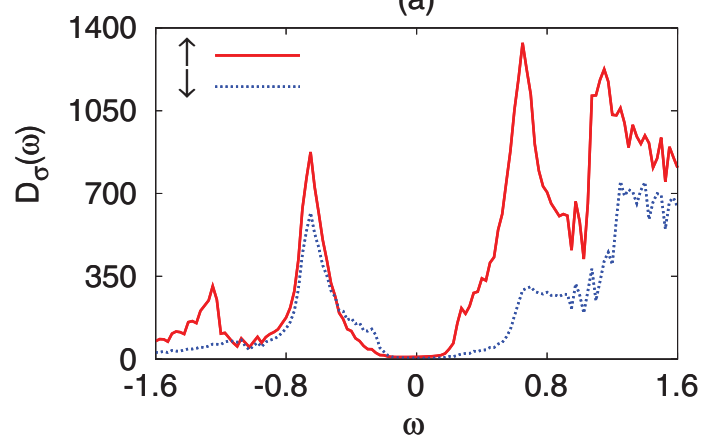

(b)

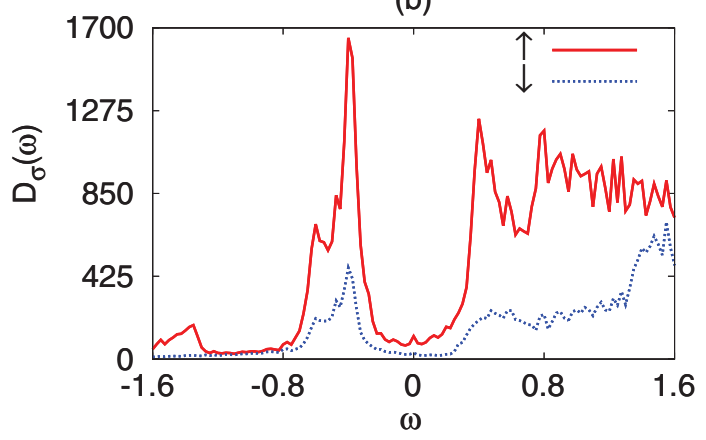

FIG. 5. (Color online) The integrated density of $\sigma$ states $D_{\sigma}(\omega)$ (in units of $1 / \varepsilon_{F}$ ) are shown as a function of energy $\omega$ (in units of $\varepsilon_{F}$ ), for the parameters considered in Fig. 3 .

particular, for a vortex line, and after using the orthonormality conditions for the Bessel functions, $D_{\sigma}(\omega)$ reduces to

$$
D_{\sigma}(\omega)=\sum_{n m s j}\left[\left(c_{n m s j}^{\sigma}\right)^{2} \delta\left(\omega-\varepsilon_{n m s}\right)+\left(d_{n m s j}^{\sigma}\right)^{2} \delta\left(\omega+\varepsilon_{n m s}\right)\right] .
$$

We use a small spectral broadening $\left(0.01 \varepsilon_{F}\right)$ to regularize the $\delta$ functions in our numerical calculations.

In Fig. 5, the integrated density of $\sigma$ states $D_{\sigma}(\omega)$ are shown as a function of energy $\omega$, for the parameters considered in Fig. 3. We see that while the density of states vanishes around $\omega=0$ in the SF phase, due to the presence of a gap in the energy spectrum, it is finite in the GSF phase with very small peaks around $\omega=0$. These peaks are due to the presence of discrete core and edge states within the continuum gap in energy, and they are most clearly seen in the majority $(\uparrow)$ component. We also note that the appearance of a second continuum branch in the excitation spectrum increases the density of states considerably when $\left|\varepsilon_{n m s}\right| \gtrsim 1.2 \varepsilon_{F}$. Next, we analyze the local probability-current density of $\sigma$ fermions, which also shows signatures for the edge-bound and Majorana zero-energy bound states.

\section{Probability-current density}

Similar to the usual $\alpha=0$ treatment, the quantum mechanical probability-current operator for $\sigma$ fermions can be identified from the continuity equation. While the presence of a spin-orbit coupling leads to additional terms in the total particle current operator, these terms do not contribute to the current since the expectation value $\left\langle\psi_{\uparrow}^{\dagger}(\mathbf{r}) \psi_{\downarrow}(\mathbf{r})\right\rangle=0$. 


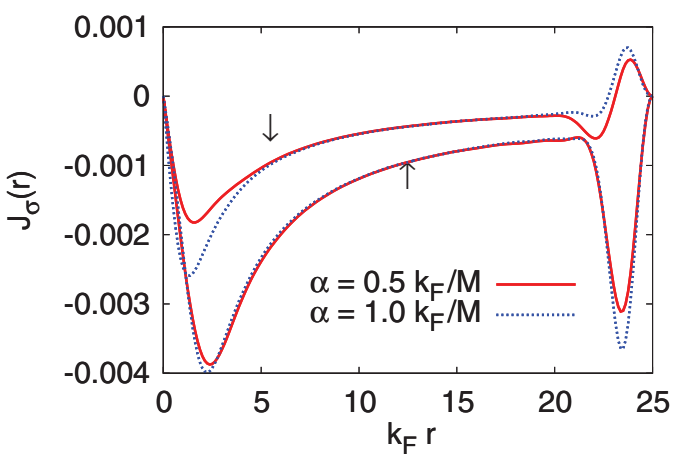

FIG. 6. (Color online) The probability-current density of $\sigma$ fermions (in units of $M / k_{F}^{3}$ ) are shown as a function of radial distance $r$ (in units of $1 / k_{F}$ ), for the parameters considered in Fig. 2.

Therefore, using the Bogoliubov-Valatin transformations, the local current density $\mathbf{J}_{\sigma}(\mathbf{r})=\left[1 /\left(2 M_{\sigma} i\right)\right]\left\langle\psi_{\sigma}^{\dagger}(\mathbf{r}) \nabla \psi_{\sigma}(\mathbf{r})-\right.$ H.c.) circulating around a single vortex line becomes $\mathbf{J}_{\sigma}(\mathbf{r})=\left[1 /\left(2 M_{\sigma} i\right)\right] \sum_{n}\left[u_{n}^{\sigma *}(\mathbf{r}) \nabla u_{n}^{\sigma}(\mathbf{r}) f\left(\varepsilon_{n}\right)+v_{n}^{\sigma *}(\mathbf{r})\right.$ $\nabla v_{n}^{\sigma}(\mathbf{r}) f\left(-\varepsilon_{n}\right)-$ H.c.], where H.c. is the Hermitian conjugate. Since $\mathbf{J}_{\sigma}(\mathbf{r})$ circulates along the $\widehat{\theta}$ direction, i.e., $\mathbf{J}_{\sigma}(\mathbf{r})=J_{\sigma}(r) \widehat{\theta}$, we find

$$
\begin{aligned}
J_{\uparrow}(r)= & \frac{1}{2 \pi M_{\uparrow} r} \sum_{n m s}\left\{m\left[\sum_{j} c_{n m s j}^{\uparrow} \phi_{j, m}(r)\right]^{2} f\left(\varepsilon_{n m s}\right)\right. \\
& \left.-(m+2)\left[\sum_{j} d_{n m s j}^{\uparrow} \phi_{j, m+2}(r)\right]^{2} f\left(-\varepsilon_{n m s}\right)\right\}, \quad(7) \\
J_{\downarrow}(r)= & \frac{1}{2 \pi M_{\downarrow} r} \sum_{n m s}\left\{(m+1)\left[\sum_{j} c_{n m s j}^{\downarrow} \phi_{j, m+1}(r)\right]^{2} f\left(\varepsilon_{n m s}\right)\right. \\
& \left.-(m+1)\left[\sum_{j} d_{n m s j}^{\downarrow} \phi_{j, m+1}(r)\right]^{2} f\left(-\varepsilon_{n m s}\right)\right\}
\end{aligned}
$$

for the strengths of the particle-current densities.

In Fig. 6, the probability-current density of $\sigma$ fermions are shown as a function of radial distance $r$, for the parameters considered in Fig. 2. When $\alpha=0$, the core-bound states have a negative (diamagnetic) and the continuum states have a positive (paramagnetic) contribution to $J_{\sigma}(r)$. This leads to a nonmonotonic $J_{\sigma}(r)$, which first increases as $\propto r$ and then decreases as $\propto 1 / r$ [27]. The latter behavior is due to the saturation of the superfluid density for long distances away from the vortex core. Therefore, a maximum peak current occurs at some distance away from the vortex core. When $\alpha \neq 0$, the major difference is at the edge. The $\uparrow$ and $\downarrow$ currents flow in opposite directions, and their magnitude increases with increasing $\alpha$. Such a counterflow of mass currents occurs even for the SF phase (not shown). Since the sums in Eqs. (7) and (8) are over states with $\varepsilon_{n m s} \geqslant 0$, counterflowing edge currents result from the asymmetry of the energy spectrum around $m=0$, due to the presence of edge states.

\section{CONCLUSIONS}

To conclude, we considered a Rashba-type spin-orbit coupled Fermi gas, and used the BdG formalism to analyze a single vortex line along a finite cylinder with a periodic boundary condition. When the populations of the pseudospin components are sufficiently imbalanced, depending on the strength of the spin-orbit coupling, we showed that coreand edge-bound states, as well as Majorana zero-energy quasiparticles, appear in the energy spectrum. These states leave signatures in the density of single-particle states and particle-current density, and particularly, we found that the pseudospin components counterflow near the edge of the cylinder, the strength of which increases with increasing spin-orbit coupling.

While preparing this work, we became aware of a closely related work [31] where the vortex core and edge states are analyzed for a trapped two-dimensional Fermi gas. For the most part, our work is consistent with their findings. However, in contrast to our finite-cylinder setup where the system is either in an SF or GSF phase, depending on the parameter regime, both SF and GSF phases may also coexist in a trap in different regions. The possibility of such a phase coexistence again leads to Andreev scattering at the SF-GSF phase boundary, giving rise to an additional branch of edge-bound states.

\section{ACKNOWLEDGMENTS}

This work is supported by the Marie Curie International Reintegration (Grant No. FP7-PEOPLE-IRG-2010-268239), Scientific and Technological Research Council of Turkey (Career Grant No. TÜBITAK-3501-110T839), and the Turkish Academy of Sciences (TÜBA-GEBIP).
[1] Y.-J. Lin, R. L. Compton, A. R. Perry, W. D. Phillips, J. V. Porto, and I. B. Spielman, Phys. Rev. Lett. 102, 130401 (2009).

[2] Y.-J. Lin, Y.-J. Lin, K. Jiménez-García, and I. B. Spielman, Nature (London) 471, 83 (2011).

[3] M. Gong, S. Tewari, and C. Zhang, Phys. Rev. Lett. 107, 195303 (2011); G. Chen, M. Gong, and C. Zhang, Phys. Rev. A (to be published).

[4] J. P. Vyasanakere, S. Zhang, and V. B. Shenoy, Phys. Rev. B 84, 014512 (2011)
[5] Z. Q. Yu and H. Zhai, Phys. Rev. Lett. 107, 195305 (2011); H. Zhai, e-print arXiv:1110.6798.

[6] Hui Hu, L. Jiang, X.-J. Liu, and Han Pu, Phys. Rev. Lett. 107, 195304 (2011); Phys. Rev. A 84, 063618 (2011).

[7] M. Iskin and A. L. Subaşi, Phys. Rev. Lett. 107, 050402 (2011); Phys. Rev. A 84, 043621 (2011).

[8] W. Yi and G.-C. Guo, Phys. Rev. A 84, 031608(R) (2011).

[9] K. Seo, Li Han, and C. A. R. Sáde Melo, e-print arXiv:1108.4068; e-print arXiv:1110.6364.

[10] L. He and X. G. Huang, e-print arXiv:1109.5577 (2011). 
[11] S. K. Ghosh, J. P. Vyasanakere, and V. B. Shenoy, Phys. Rev. A 84, 053629 (2011).

[12] B. Huang and S. Wan, e-print arXiv:1109.3970; X. Yang and S. Wan, e-print arXiv:1111.4277.

[13] J. Zhou, W. Zhang, and W. Yi, Phys. Rev. A 84, 063603 (2011).

[14] J. N. Zhang. Y. H. Chan, and L. M. Duan, e-print arXiv:1110.2241.

[15] K. Zhou and Z. Zhang, e-print arXiv:1110.3565.

[16] R. Liao, Y. Y. Xiang, and W.-M. Liu, e-print arXiv:1110.5818.

[17] G. Moore and N. Read, Nucl. Phys. B 360, 362 (1991).

[18] G. Volovik, JETP Lett. 70, 609 (1999).

[19] N. Read and D. Green, Phys. Rev. B 61, 10267 (2000).

[20] S. Tewari, S. Das Sarma, and D.-H. Lee, Phys. Rev. Lett. 99, 037001 (2007).

[21] T. Mizushima, M. Ichioka, and K. Machida, Phys. Rev. Lett. 101, 150409 (2008).

[22] L. Fu and C. L. Kane, Phys. Rev. Lett. 100, 096407 (2008).

[23] Y. Oreg, G. Refael, and F. von Oppen, Phys. Rev. Lett. 105, 177002 (2010).
[24] J. Alicea, Y. Oreg, G. Refael, F. von Oppen, and M. P. A. Fisher, Nature Phys. 7, 412 (2011).

[25] J. D. Sau, R. M. Lutchyn, S. Tewari, and S. Das Sarma, Phys. Rev. Lett. 104, 040502 (2010).

[26] Li Mao and C. Zhang, Phys. Rev. B 82, 174506 (2010).

[27] F. Gygi and M. Schlüter, Phys. Rev. B 43, 7609 (1991).

[28] M. Takahashi, T. Mizushima, M. Ichioka, and K. Machida, Phys. Rev. Lett. 97, 180407 (2006)

[29] C. Caroli, P. de Gennes, and J. Matricon, Phys. Lett. 9, 307 (1964).

[30] This is similar to what happens for a single vortex in twodimensional chiral $p$-wave superfluids [21], where Majorana core- and edge-bound states also appear in the weakly interacting BCS regime.

[31] X.-J. Liu, L. Jiang, Han Pu, and Hui Hu, e-print arXiv:1111.1798. Our formalism and theirs have minor differences, e.g., $v_{n}^{\sigma}(\mathbf{r})$ amplitudes differ by a minus sign in their Bogoliubov-Valatin transformation, leading to some other presentational differences. 